\title{
Treating relapsed or refractory Philadelphia chromosome-negative acute lymphoblastic leukemia: liposome-encapsulated vincristine
}

This article was published in the following Dove Press journal:

International Journal of Nanomedicine

12 September 2013

Number of times this article has been viewed

\section{Tyler Davis}

Sherif S Farag

Department of Internal Medicine, Division of Hematology and Oncology, Indiana University School of Medicine, Indianapolis, IN, USA
Correspondence: Sherif S Farag Department of Internal Medicine, Indiana University School of Medicine, R3-Walther Hall, C4I4, 980 West Walnut Street, Indianapolis, IN 46202, USA

Tel +l 3172780490

Email ssfarag@iu.edu

\begin{abstract}
Acute lymphoblastic leukemia (ALL) remains a disease with poor outcomes in adults. While induction chemotherapy achieves a complete remission in almost $90 \%$ of patients, the majority will relapse and die of their disease. Relapsed ALL is associated with a high reinduction mortality and chemotherapy resistance, with allogeneic hematopoietic stem cell transplantation offering the only therapy with curative potential. However, there is no efficacious and well tolerated standard regimen accepted as a "bridge" to allogeneic stem cell transplantation or as definitive treatment for patients who are not transplant candidates. Vincristine is an active drug in patients with ALL, but its dose intensity is limited by neurotoxicity, and its full potential as an anticancer drug is thus not realized. Encapsulation of vincristine into sphingomyelin and cholesterol nanoparticle liposomes facilitates dose-intensification and densification to enhanced target tissues with reduced potential for toxicity. Vincristine sulfate liposome injection (VSLI) is associated with significant responses in clinically advanced ALL, and has recently been approved by the US Food and Drug Administration for treatment of relapsed and clinically advanced Philadelphia chromosome-negative ALL. This review provides an overview of the preclinical and clinical studies leading to the approval of VSLI for the treatment of relapsed and refractory ALL, and suggests potential areas of future clinical development.
\end{abstract}

Keywords: vincristine, lymphoblastic leukemia, liposome

\section{Introduction}

Acute lymphoblastic leukemia (ALL) is a heterogeneous group of malignancies of committed precursor lymphoid cells characterized by the clonal proliferation of T-cell or B-cell lineage lymphoblasts. ALL is primarily a disease of children, with a peak incidence of 7.7 per 100,000 between the ages of one and 4 years. Sixty percent of ALL cases are diagnosed before 20 years of age. ${ }^{1}$ While the rate begins to decline after the first decade of life, the incidence increases again in the fifth decade of life, with a smaller peak observed in patients older than 85 years. $^{2}$ In adults, approximately $30 \%$ of ALL cases are associated with a biologically and clinically distinct variant characterized by the Philadelphia chromosome, a reciprocal translocation between chromosomes 9 and 22, designated as $\mathrm{t}(9 ; 22)(\mathrm{q} 34 ; \mathrm{q} 11.2)$, which results in a constitutively active fusion tyrosine kinase protein, BCR-ABL. ${ }^{3,4}$ In contrast with children with ALL, the prognosis for adults diagnosed with ALL remains poor. ${ }^{2,5-7}$ Compared with children with ALL, in whom long-term survival approaches $90 \%,{ }^{8}$ the leukemia-free survival of adults with ALL ranges from 30\% to 40\% in large series with 3-7 years of follow-up..$^{9-11}$ Despite an initial complete remission rate of up to $90 \%$ following induction chemotherapy, a majority of adults will relapse and die of ALL., ${ }^{9,12}$ The high 
rate of relapse in adults with ALL as compared with children likely relates to many factors, including decreased response to regimens less toxic than pediatric induction chemotherapy regimens. ${ }^{14-16}$ For patients who relapse after initial induction chemotherapy, allogeneic hematopoietic stem cell transplantation (HSCT) remains the best treatment option, although only a minority of patients makes it to transplant due to disease resistance, toxicity of salvage therapy, and comorbidities. ${ }^{7}$ However, in such patients, achievement of a second complete remission is a priority for optimal long-term outcome following allogeneic transplantation. Currently, there is no uniformly accepted standard salvage treatment for relapsed ALL, and novel therapies to improve outcome without increasing toxicities are required.

Recently, there has been growing interest in liposomeencapsulated drugs for delivery of more efficacious treatment with less toxicity. The clinical utility of most conventional chemotherapeutics is limited either by the inability to deliver therapeutic drug concentrations to the target tissues or by severe and harmful toxic effects on normal organs and tissues. ${ }^{17}$ Liposome-encapsulated drugs represent a potential way to overcome these limitations. Liposomes are small, spherical, and enclosed compartments separating an aqueous medium from another biphospholipid bilayer. ${ }^{17}$ Figure 1 illustrates how drugs can be packaged for delivery to target tissues within a liposome. Liposomes were first discovered by the British hematologist, Alec Bangham, ${ }^{18}$ and the first liposomal pharmaceutical product, liposomal doxorubicin (Doxil $^{\circledR}$, Johnson \& Johnson, Brunswick, NJ, USA), received United States Food and Drug Administration (FDA) approval in 1995 for the treatment of chemotherapy-refractory acquired immune deficiency syndrome-related Kaposi's sarcoma. ${ }^{17}$ Doxil is currently approved for use in recurrent ovarian cancer and in relapsed or refractory multiple myeloma. Given the activity of liposome-encapsulated doxorubicin and other drugs, ${ }^{17}$ investigators have looked at additional drugs with activity in ALL that could also be encapsulated.

Vincristine, like the other vinca alkaloids, is very active against many of the lymphoid malignancies, including aggressive non-Hodgkin's lymphoma and ALL. ${ }^{19}$ In adults with ALL, vincristine remains an integral component of induction chemotherapy regimens. ${ }^{20-23}$ Vincristine acts by binding to tubulin during active mitosis, resulting in microtubule depolymerization and metaphase arrest, leading to apoptosis. ${ }^{24,25}$ However, vincristine also binds to neuronal tubulin, disrupts axonal microtubules, and thus causes severe neurotoxicity, which has led to the clinical practice of capping the total dose of vincristine to $2.0 \mathrm{mg}$ regardless of body surface area, potentially reducing clinical efficacy. The high affinity of vincristine to both mitotic and neuronal microtubules suggests that it may be difficult to prevent neurotoxicity without reducing clinical efficacy. ${ }^{26}$ The dosing limitations of vincristine have led researchers to investigate the use of liposomes to deliver vincristine with an increased half-life of

A
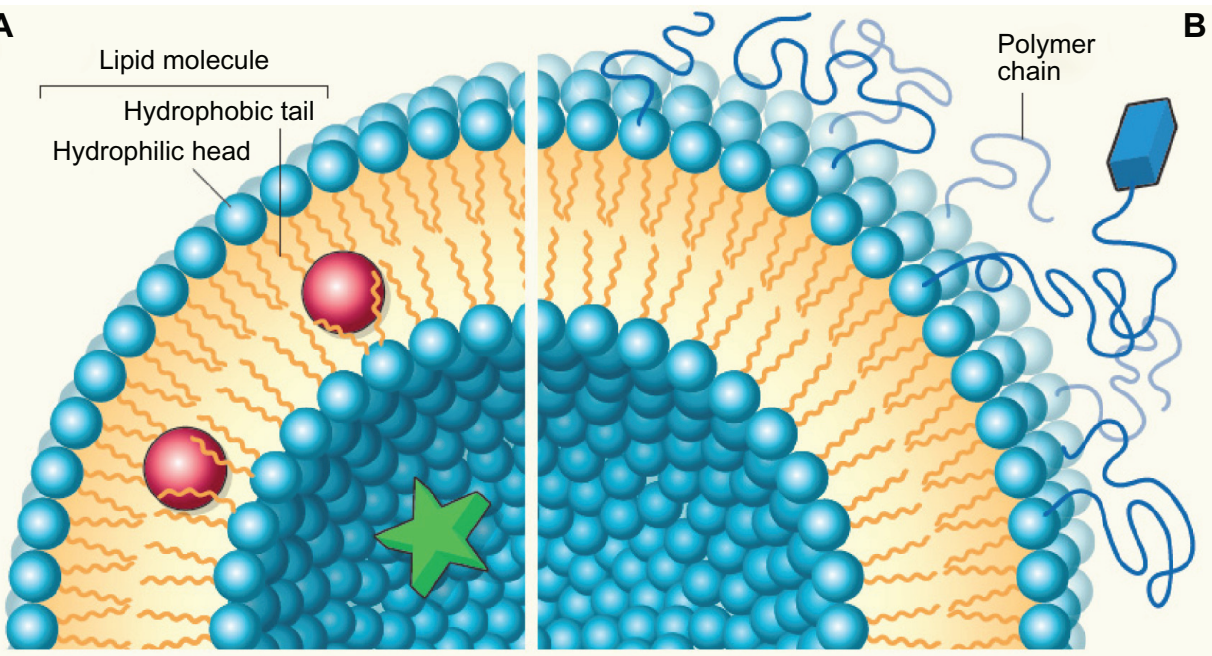

Figure I Liposomes for drug delivery. Liposomes are spherical vesicles that have a lipid bilayer shell and can be modified for delivery of drugs. (A) Simple liposomes can trap hydrophobic drug molecules a few nanometers in diameter (red spheres) within the hydrophobic bilayer, and hydrophilic molecules up to several nanometers (green star) in its larger interior. (B) "Stealth" liposomes contain a small percentage of water-soluble polymers (eg, polyethylene glycol) chemically attached to the polar heads of lipids to mitigate rapid removal of the liposome by immune cells in vivo, and are better suited to drug delivery. Peptides (blue rectangle) that target specific biological targets may be attached to the polymers. While polymers have been employed in liposome encapsulation of some anticancer drugs, such as doxorubicin, they are not currently used in liposomal vincristine formulations.

Note: Adapted by permission from Macmillan Publishers Ltd: Nature. 20I2;489(74I6):372-374. Safinya and Ewert. Materials chemistry: Liposomes derived from molecular vases. Copyright (C) $2012 .^{27}$ 
the drug while decreasing toxicity. Liposome-encapsulated vincristine appears to be less neurotoxic in animal models as well as in humans. ${ }^{26}$ This review discusses current therapies for relapsed Philadelphia chromosome-negative ALL, focusing on liposome-encapsulated vincristine as a novel approach to a potentially more efficacious and safer means of delivering the drug.

\section{Treatment of relapsed Philadelphia-negative ALL}

From a therapeutic perspective, it is useful to subclassify ALL according to the presence or absence of the Philadelphia chromosome. While Philadelphia chromosome-positive ALL is associated with a poor prognosis, the use of molecularly targeted therapy with imatinib or other tyrosine kinase inhibitors, in combination with chemotherapy, has improved the outcome of this disease variant. ${ }^{28-31}$ Currently, there are no similar molecularly targeted agents approved for the treatment of Philadelphia chromosome-negative ALL variants. Allogeneic HSCT may improve the survival of adults when performed in first complete remission, ${ }^{32}$ and remains the best curative modality for patients with relapsed disease. ${ }^{33-35}$ While the vast majority of adults with ALL will achieve complete remission with primary induction chemotherapy, most will relapse.

The goal of therapy in relapse is to obtain a remission without undue toxicity and to move to allogeneic transplantation as rapidly as possible if the patient is eligible. However, a vast majority of ALL patients, who are otherwise eligible for transplantation, die shortly after relapsing, and many do not achieve a second complete remission. ${ }^{36}$ This is emphasized by a study of 609 relapsed patients where the overall survival at 5 years was only $7 \%,{ }^{12}$ highlighting the need for improved treatment of relapsed disease. Currently, there is no uniformly accepted standard of care for relapse, and outcomes with salvage chemotherapy for adults with relapsed or refractory ALL have traditionally been poor. ${ }^{5}$

Multiple salvage chemotherapy regimens are used in relapsed ALL. ${ }^{37}$ Salvage regimens typically can be grouped according to the backbone drugs in a combination, and include: vincristine, corticosteroids, and anthracyclinebased combinations; asparaginase-based combinations with or without methotrexate; high-dose cytarabine-based combinations; and miscellaneous combinations or single agents, including clofarabine and nelarabine (particularly for T-lineage ALL). Currently no survival benefit is apparent with any given chemotherapy approach, ${ }^{12}$ with drug resistance being a major limitation to successful treatment. As noted above, while intolerance of adult patients to high-intensity pediatric regimens may be partly responsible for the higher relapse rate, molecular and biological mechanisms of drug resistance continue to be described. Recently, activating mutations of the gene $N T 5 C 2$, encoding the enzyme cytosolic purine $5^{\prime}$-nucleotidase responsible for inactivating the nucleoside analogs 6-mercaptopurine and 6-thioguanine, which are commonly used in ALL treatment, have been shown to be present in a small proportion of B-lineage and T-lineage ALL. ${ }^{38,39}$ In addition, multiple preclinical studies have identified abnormal cellular signaling in leukemia cells as a possible mechanism. For example, the immunoglobulin heavy chain binding protein, BIP/HSPA5, a pivotal component of the prosurvival axis of the unfolded protein response signaling network, is abundantly expressed in relapsed B-cell ALL and has been proposed as a chemotherapy-resistance biomarker. ${ }^{40} \mathrm{~A}$ murine model also detailed that obesity might be a factor in resistance, with ALL cells preferentially migrating to a proposed protective microenvironment within adipose cells. ${ }^{41}$ A number of Phase I and II clinical trials of novel agents are currently underway to investigate novel targeted therapies for relapsed ALL to improve outcomes. ${ }^{42,43}$ An alternative approach is to alter the delivery of drugs known to be efficacious against the disease to enhance drug exposure while minimizing limiting toxicity. Use of nanoparticle technology to enhance delivery of vincristine is one such promising therapy in ALL.

Vincristine is an important and active drug in first-line therapy of ALL as well as in the treatment of relapsed disease. ${ }^{44}$ Its administration is generally limited to weekly injections, usually for 2 months or less in most regimens because of severe peripheral neuropathy, including autonomic neuropathy that often leads to intestinal complications. Because of its narrow therapeutic index, the maximal antileukemia activity of free vincristine is not realized. ${ }^{24}$ Thus, an approach to increasing the dose intensity of vincristine while decreasing its toxicity through the use of liposome encapsulation technology has been investigated.

\section{Liposome-encapsulated anticancer drugs}

Liposomes are artificially prepared vesicles composed of a lipid bilayer that are used as drug delivery vehicles, and have been shown to enhance the therapeutic activity of a number of anticancer drugs. ${ }^{17,45-50}$ Liposomes encapsulate a region of aqueous solution inside a hydrophobic membrane (Figure 1), and can be used to overcome suboptimal pharmaceutical properties of certain drugs, including low solubility, 
instability, and rapid metabolism, and may reduce systemic toxicity by allowing selective delivery of the drug to the tumor site. The extent of encapsulation of a dissolved aqueous drug, such as vincristine sulfate, and subsequent retention of the drug within the liposome, both in vitro and in vivo, has been shown to depend on the lipid composition of the liposome and on the magnitude of the transmembrane $\mathrm{pH}$ gradient. ${ }^{51}$ Multiple lipids have been used to construct liposomes. Most utilize a phosphatidylglycerol or phosphatidylcholine base, while some are derived from egg or soy. ${ }^{17}$ Doxil is a PEGylated liposome using distearoylphosphatidylethanolamine bound to polyethylene glycol. ${ }^{52}$ Liposomes containing a low $\mathrm{pH}$ can be constructed such that dissolved aqueous drugs will be charged in solution, and because the $\mathrm{pH}$ naturally neutralizes within the liposome through transmembrane passage of protons, the drug will also be neutralized, allowing it to pass freely through the membrane.

Appropriately designed liposomes also have the ability to accumulate passively in tissues with fenestrated vasculature (eg, bone marrow, spleen, liver, lymph nodes, and within solid tumors), ${ }^{53}$ resulting in enhanced exposure of tumor tissue to drug to a greater extent than can be achieved by administration of free drug. Also, retention of the drug in the liposome can increase its duration of exposure to cancer cells, while minimizing the concentration of free drug in the circulation, which can contribute to toxicity. ${ }^{54}$

The earliest work focused on liposomal encapsulation of anthracyclines, ${ }^{45,46}$ eventually leading to approval of liposomeencapsulated doxorubicin (Doxil) by the FDA, and subsequently liposome-encapsulated daunorubicin (DaunoXome ${ }^{\circledR}$, Galen Pharmaceuticals, Craigavon, Northern Ireland). In both cases, the liposomal anthracycline formulation retained the efficacy of the free drug but was associated with reduced toxicity in clinical trials in a variety of cancers, ${ }^{55-58}$ including ALL, where the use of liposomal daunorubicin in place of free doxorubicin in the hyper-CVAD (fractionated cyclophosphamide, vincristine, doxorubicin, and dexamethasone) regimen was well-tolerated in relapsed or refractory ALL. ${ }^{57}$ The development of these two drugs helped to establish the paradigm for approval that if a liposomal-encapsulated drug could be shown to be comparable in efficacy to its free counterpart while causing less adverse events, then it could be approved for use.

Liposomal cytarabine $\left(\right.$ Depocyt $^{\circledR}$, Pacira Pharmaceuticals Inc, San Diego, CA, USA) is another drug active in ALL which, after encapsulation, showed improved outcomes when used intrathecally to treat leptomeningeal disease. ${ }^{59}$ The systemic use of liposomal cytarabine was investigated in a Phase I study using a fixed molar ratio formulation of cytarabine and daunorubicin (CPX-351) in 48 patients with relapsed or refractory acute myeloid leukemia or high-risk myelodysplastic syndrome. ${ }^{60}$ Pharmacokinetic data showed that the intended plasma ratio of 5:1 was maintained at all dose levels, while nearly all of the detectable cytarabine and daunorubicin in the circulation was liposome-encapsulated. ${ }^{61}$ Although not tested in ALL, a randomized Phase II trial is currently ongoing using CPX-351 for the treatment of high-risk patients with untreated myelodysplastic syndrome or acute myeloid leukemia, and is attempting to improve the treatment-related mortality rate while at least keeping the complete remission rate constant (ClinicalTrials.gov identifier, NCT01804101). These studies continue to support further investigation of drugs active in hematologic malignancies in newly developed liposome-encapsulated versions.

\section{Liposome-encapsulated vincristine}

Vincristine is a vinca alkaloid with activity against a broad range of cancers, including Hodgkin's and non-Hodgkin's lymphoma, chronic lymphocytic leukemia, ALL, and a variety of solid tumors. As noted above, vincristine exerts its cytotoxic activity by attaching to the growing end of microtubules and preventing their assembly, arresting cell growth in metaphase. Therefore, as a cell-cycle specific cytotoxic drug, prolonging exposure time to the drug is expected to be therapeutically advantageous; perhaps even more so than prolonged exposure to anthracyclines which do not act in a cell cycle-specific manner. In leukemia cell lines, exposure to vincristine from one to 72 hours resulted in a $10^{5}$-fold reduction in the drug concentration needed to cause $50 \%$ cytotoxicity $\left(\mathrm{IC}_{50}\right)$, compared with only a 40 -fold reduction in the $\mathrm{IC}_{50}$ for doxorubicin following prolonged exposure to the same extent. ${ }^{48,51}$ Pharmacokinetic data with free vincristine detailed rapid tissue binding, low serum concentrations after intravenous administration, and extensive tissue distribution in vivo, ${ }^{62,63}$ limiting its potential therapeutic efficacy. While continuous intravenous infusion of vincristine has been investigated as a way to increase drug exposure, significant neurotoxicity still occurred. ${ }^{64}$ Furthermore, conventional vincristine is limited by significant peripheral and central nervous system neurotoxicity, which occurs at doses higher than $1.4 \mathrm{mg} / \mathrm{m}^{2}$. Therefore, it was hypothesized that if liposomal encapsulation and delivery results in higher levels of drug at tumor sites for longer periods of time, greatly improved efficacy may be expected with a cell cycle-specific drug like vincristine, ${ }^{51}$ and retention of drug in the liposome 
would result in lower drug concentrations in tissues where toxicity occurs, including the peripheral and central nervous systems. $^{24}$

\section{Preclinical development of liposome- encapsulated formulations of vincristine}

The earliest investigations of liposomal encapsulation of vincristine failed to demonstrate a therapeutic advantage over free vincristine sulfate in murine leukemia models. ${ }^{65}$ Indeed, the low solubility of vincristine in aqueous solution at physiologic $\mathrm{pH}$ and its relatively high permeability to membranes, resulting in poor retention of drug in the liposome, initially presented significant limitations to development of a stable liposomal vincristine formulation. Subsequent work identified that transmembrane $\mathrm{pH}$ gradients, with the inside of the vesicle being acidic, can result in significantly improved trapping of drug in the liposome. ${ }^{66-68}$ Furthermore, maintenance of the $\mathrm{pH}$ gradient is affected, at least in part, by the lipid composition of the liposome. ${ }^{47}$ Utilizing such transmembrane $\mathrm{pH}$ gradient drug uptake processes, almost 100\% trapping of vincristine was achieved inside egg phosphatidylcholine/cholesterol and distearoylphosphatidylcholine (DSPC)/cholesterol liposomes, when the internal $\mathrm{pH}$ of the liposome was lowered to 4.0. ${ }^{47}$ However, while both DSPC/cholesterol and egg phosphatidylcholine/cholesterol preparations had similar vincristine trapping efficiency, the $\mathrm{pH}$ gradient across the membrane was significantly more sustained in DSPC/cholesterol vesicles with better retention of vincristine in the liposome, ${ }^{47}$ resulting in a longer drug circulation time and enhanced tumor delivery and antitumor activity compared with free vincristine. ${ }^{47,48}$ In murine models of lymphocytic leukemia, the DSPC/cholesterol liposome formulation showed greater antitumor efficacy compared with either free vincristine or the egg phosphatidylcholine/cholesterol formulation, ${ }^{47,48}$ and the $\mathrm{LD}_{50}$ (median lethal dose) was significantly higher with DSPC/cholesterol liposome-encapsulated vincristine formulation $\left(\mathrm{LD}_{50} 4.8 \mathrm{mg}\right.$ / $\mathrm{kg}$ ) compared with free vincristine $\left(\mathrm{LD}_{50} 1.9 \mathrm{mg} / \mathrm{kg}\right)$, indicating that liposomal encapsulation increased drug delivery with less toxicity. ${ }^{47}$

The observation that the antitumor efficacy of liposomeencapsulated vincristine increases with liposome circulation time and increased retention of the drug within the liposome after intravenous administration ${ }^{47,48,69}$ led to further attempts at optimizing the liposome formulation. However, because lowering the $\mathrm{pH}$ buffer within the liposome to increase the $\mathrm{pH}$ gradient and improve vincristine retention resulted in increased acid-catalyzed hydrolysis of the phosphatidylcholine component of the liposome, more chemically stable lipids were investigated. ${ }^{69,70}$ This led to identification of sphingomyelin/ cholesterol liposomes, which were more resistant to acidmediated hydrolysis, and also had a longer in vivo circulation time compared with DSPC/cholesterol liposomes, likely related to lower adsorption of serum proteins to the sphingomyelin/cholesterol liposomes, which contributes to clearance of the liposomes by the mononuclear phagocytic system. ${ }^{70} \mathrm{In}$ mouse leukemia models, it was confirmed that sphingomyelin/ cholesterol formulations were significantly more effective at reducing tumor progression than free vincristine or vincristine encapsulated in DSPC/cholesterol formulations. ${ }^{70}$

Pharmacokinetic data also confirmed that sphingomyelin/ cholesterol liposome-encapsulated vincristine had a longer half-life (6.6 hours versus 1.36 hours), a higher area under the plasma concentration-time curve $(213 \mu \mathrm{g} \cdot \mathrm{h} / \mathrm{mL}$ versus $0.59 \mu \mathrm{g} \cdot \mathrm{h} / \mathrm{mL})$, and a smaller volume of distribution $(2.0 \mathrm{~mL}$ versus $145 \mathrm{~mL}$ ) compared with free vincristine..$^{54}$ As indicated in Figure 1B, chemically attachment of water-soluble polymers, such as polyethylene glycol, to the lipid polar heads of the liposome has been used to limit removal of liposomes by phagocytic cells in vivo. ${ }^{71,72}$ However, while the addition of polyethylene glycol to sphingomyelin/cholesterol liposomes increased liposome circulation longevity, it also resulted in significant leakage of vincristine from the liposomes, resulting in no added benefit. ${ }^{70}$

Preclinical models also confirmed that vincristine encapsulated in sphingomyelin/cholesterol liposomes accumulated preferentially at tumor sites, which correlated with antitumor activity. ${ }^{54,70,73}$ Furthermore, this was related to liposomal extravasation into the tumor rather than uptake by tumor cells of free vincristine that had been released from liposomes in the circulation. ${ }^{70}$ In a murine model with human breast cancer xenografts, sphingomyelin/cholesterol liposomeencapsulated vincristine resulted in targeted delivery of the drug, with a four-fold increase in concentration of drug in tumor tissue and a three-fold increase in bone marrow, with maintenance of significant tissue drug concentrations for several days compared with free vincristine, and without increased toxicity. ${ }^{73}$ The antitumor efficacy of sphingomyelin/ cholesterol liposome-encapsulated vincristine has also been confirmed in several preclinical murine and human tumor xenograft models, representing several cancer types, ${ }^{54,70,73-76}$ including human ALL. ${ }^{24}$ The aggregate of the above studies supports the utility of encapsulating vincristine in sphingomyelin/cholesterol liposomes to increase drug delivery while limiting release in the central blood compartment to decrease drug toxicity. A schematic of the development of liposomal vincristine is summarized in Figure 2. 


\section{Clinical efficacy of vincristine sulfate liposome injection (Marqibo ${ }^{\circledR}$ ) in ALL}

Based on the efficacy of liposome-encapsulated vincristine and the decreased toxicity observed in preclinical studies, clinical trials were initiated in humans with solid tumors and relapsed hematologic malignancies. The first open-label, dose-escalation Phase I clinical trial of liposomal vincristine was conducted using a DSPC/cholesterol liposomal formulation of vincristine sulfate (ONCO-TCS; British Columbia Cancer Agency, Vancouver, BC, Canada) in 25 patients with previously treated solid tumors. ${ }^{77}$ Patients were treated with escalating doses of vincristine from $0.5 \mathrm{mg} / \mathrm{m}^{2}$ to $2.8 \mathrm{mg} / \mathrm{m}^{2}$ intravenously over 60 minutes every 3 weeks. Dose-limiting toxicities, including myalgias, peripheral neuropathy, and constipation, were observed at the highest dose level of $2.8 \mathrm{mg} / \mathrm{m}^{2}$. The maximum tolerated dose was defined as $2.4 \mathrm{mg} / \mathrm{m}^{2}$. Overall grades $3-4$ toxicities observed across all dose levels were constipation (12\%), fatigue ( $8 \%$ ), anemia $(8 \%)$, and alopecia (8\%). One partial response was seen in a patient with pancreatic cancer, and tumor response not meeting partial response criteria was seen in two other patients. ${ }^{77}$

\begin{tabular}{|c|}
\hline \multicolumn{1}{|c|}{ Free vincristine } \\
\hline $\begin{array}{l}\text { Weaknesses of dose-limiting neurotoxicity with a } \\
\text { large volume of distribution leading to extensive } \\
\text { tissue binding }\end{array}$ \\
\hline $\begin{array}{c}\text { Distearoylphosphatidylcholine and } \\
\text { cholesterol liposomes }\end{array}$ \\
\hline $\begin{array}{l}\text { Utilized a pH gradient to enhance tumor delivery } \\
\text { with decreased toxicity versus free vincristine }\end{array}$ \\
\hline Sphingomyelin and cholesterol liposomes \\
\hline $\begin{array}{l}\text { Developed for optimum pharmacokinetics with } \\
\text { longer plasma circulation time and enhanced } \\
\text { target-tissue accumulation }\end{array}$ \\
\hline Vincristine sulfate liposomal injection \\
\hline
\end{tabular}

Figure 2 Development of vincristine sulfate liposomal injection. Free vincristine is active in acute lymphoblastic leukemia, but has suboptimal pharmacokinetics with a large volume of distribution leading to extensive tissue binding. Utilizing the knowledge that other anticancer drugs had been encapsulated in liposomes to overcome similar pharmacokinetic challenges, vincristine was encapsulated with a distearoylphosphatidylcholine and cholesterol liposomes with a pH gradient to load the drug into the vesicles. Subsequent development of liposomal formulations to optimize pharmacokinetic properties led to the identification and development of sphingomyelin/cholesterol liposomes, for encapsulation of vincristine. These sphingomyelin liposomes offer the advantages of longer plasma circulation time and enhanced target-tissue accumulation.
Pharmacokinetic studies, which measured total plasma (both liposome-entrapped and nonencapsulated) vincristine concentrations, indicated that plasma elimination of vincristine after injection best fits a two-compartment model, suggesting that this formulation protects vincristine from the early phase of rapid elimination seen with the standard nonencapsulated drug formulation. ${ }^{77,78}$ In addition, total vincristine plasma concentrations were significantly greater following liposomal administration than described previously following nonencapsulated vincristine injection. ${ }^{77,78}$ It should be noted, however, that the plasma concentration of free vincristine released from the liposome into the circulation was too low to be quantified.

Subsequent clinical investigation, including clinical development in ALL patients, was performed using the optimized liposome formulation, ie, vincristine sulfate liposome for injection (VSLI, Marqibo ${ }^{\circledR}$, Talon Therapeutics Inc, San Francisco, CA, USA). Marqibo is a proprietary nanoparticle formulation of vincristine in an aqueous core of sphingomyelin and cholesterol liposomes developed for clinical use. Evaluation of VSLI in patients with ALL followed encouraging results in 16 adult patients with relapsed and refractory ALL who were enrolled on an extension study of a Phase II trial in patients with relapsed, aggressive nonHodgkin's lymphoma. ${ }^{26}$ Eight of these patients had Philadelphia chromosome-positive ALL. Patients received VSLI $2 \mathrm{mg} / \mathrm{m}^{2}$ intravenously over 60 minutes every 2 weeks until rapid disease progression or dose-limiting toxicity occurred. Of 14 patients evaluable for response, two responded (one complete remission, one partial response) and four further patients had transient reduction in bone marrow blasts. Toxicity was minimal, although treatment was limited, with only a median two (range 1-5) doses delivered. Two patients developed grade 1 peripheral neuropathy after two and four doses, respectively, and one patient developed grade 3 seizure that was not attributed to VSLI. ${ }^{79}$ The observed antileukemic activity of VSLI in this refractory group of patients, and the minimal toxicity observed, warranted further investigation of this agent in relapsed ALL.

To optimize dosing and better define the toxicity of VSLI in adult patients with relapsed or refractory ALL, a multicenter Phase I trial was conducted to determine the maximum tolerated dose. ${ }^{80}$ Thirty-six patients of median age 32 years with relapsed or refractory ALL, all pretreated with conventional vincristine, received at least one dose of VSLI. In a $3+3$ dose-escalation design, five dose levels of intravenous VSLI were tested, including $1.5 \mathrm{mg} / \mathrm{m}^{2}, 1.825 \mathrm{mg} / \mathrm{m}^{2}, 2.0 \mathrm{mg} / \mathrm{m}^{2}, 2.25 \mathrm{mg} / \mathrm{m}^{2}$, and 
$2.4 \mathrm{mg} / \mathrm{m}^{2}$. VSLI was administered weekly, together with dexamethasone (40 mg) on days 1-4 and 11-14 of each 4-week cycle, with therapy continued until progression or dose-limiting toxicity. The maximum tolerated dose of VSLI was $2.25 \mathrm{mg} / \mathrm{m}^{2}$ based on dose-limiting toxicities of grade 3 motor neuropathy, grade 4 seizure, and grade 4 hepatotoxicity in one patient each at the $2.4 \mathrm{mg} / \mathrm{m}^{2}$ dose level. The most common toxicities attributed to VSLI included peripheral neuropathy (55\%) and constipation (53\%). Seven of $36(19 \%)$ patients achieved a complete remission based on intent-to-treat analysis, and four of 14 (29\%) patients receiving VSLI as their first salvage attempt achieved complete remission. Additionally, four of the seven patients who achieved complete remission underwent subsequent allogeneic HSCT in remission. ${ }^{80}$ The results indicated that VSLI plus dexamethasone is an effective salvage treatment for patients for relapsed or refractory ALL, and may be used as a well-tolerated, successful option as a "bridge" to allogeneic transplantation.

Based on the encouraging activity in the Phase I trial, and the clear unmet need of patients with relapsed or refractory ALL, for whom no accepted standard therapy exists, a multinational, pivotal, single-arm, open-label, Phase II trial of weekly VSLI monotherapy was conducted in patients with relapsed and refractory B-cell or T-cell lineage Philadelphia chromosome-negative ALL. The results of this trial have recently been reported, ${ }^{81}$ and have served as the basis for accelerated approval of VSLI in the US by the FDA on August 9, 2012. Sixty-five adult patients, of median age 31 (range 19-83) years, with clinically advanced and heavily pretreated relapsed or refractory ALL, received intravenous VSLI $2.25 \mathrm{mg} / \mathrm{m}^{2}$ weekly on days $1,8,11$, and 22 of each 28-day cycle until disease progression, toxicity, or a decision to pursue other treatment, such as allogeneic HSCT, was made. Most patients had a large disease burden at the time of study treatment, with median bone marrow or peripheral blood blast content of $82 \%$, and more than half of the patients had received at least three lines of prior therapy. Forty-five percent of the patients were also refractory to their immediate prior line of therapy. After a median of four (range 1-18) doses of VSLI, a complete remission (or complete remission with incomplete count recovery [CRi]) was observed in $20 \%$ of patients, and an additional $15 \%$ achieved a partial response for an overall response rate of $33 \%$. Of note, eight patients who achieved complete remission/CRi were also in molecular remission by minimal residual disease assessment. The median duration of complete remission/CRi was 23 (range 5-66) weeks. Importantly, 12 of the 13 patients who achieved complete remission/CRi were successfully "bridged" to allogeneic HSCT. Thirty-seven patients (58\%) experienced at least one grade 3 or 4 treatment-related adverse event. Grade 3 peripheral neuropathy occurred in 15 patients, and one patient developed grade 4 neuropathy. Other serious adverse events included febrile neutropenia $(5 \%)$, tumor lysis syndrome (5\%), and constipation $(3 \%)$. The 30-day induction mortality was $12 \%$.

The results of the pivotal Phase II trial of VSLI monotherapy compare well with other salvage therapies in patients with clinically advanced relapsed or refractory ALL. Unfortunately, no randomized, comparative Phase III trials in this population have been reported. Currently, only limited comparisons with small studies of other agents in comparable populations can be made. In a retrospective analysis of a subgroup of 56 patients with clinically advanced relapsed and refractory Philadelphia chromosome-negative ALL treated with non-VSLI single agents, only $4 \%$ achieved complete remission/CRi, with 17 of 56 patients dying within 30 days of treatment. ${ }^{5,82}$ Single-agent clofarabine induced complete remission in only one of eight relapsed ALL patients (13\%) in need of third-line therapy, with all remission being short-lived. ${ }^{83}$ In a study of the combination of clofarabine plus cytarabine, three of $16(19 \%)$ ALL patients in need of third-line or higher treatment achieved complete remission/ CRi. ${ }^{84}$ Finally, single-agent nelarabine was reported to result in complete remission in five of 28 patients with relapsed or refractory T-cell lineage ALL or lymphoblastic lymphoma. ${ }^{85}$

\section{Conclusion}

VSLI represents a significant advance in nanotechnology for liposomal delivery of chemotherapy drugs to improve efficacy and reduce toxicity. Currently, VSLI is approved by the FDA for treatment of Philadelphia chromosome-negative ALL in second or greater relapse or where disease has progressed following two or more chemotherapy regimens. However, despite the significant clinical antileukemic activity seen in the pivotal Phase II trial that led to its approval, the role of VSLI remains unclear in ALL. Phase III trials need to be completed to demonstrate improvements in overall survival or rate of complete remission when compared with other second or third salvage regimens to establish fully the role of liposomal vincristine in Philadelphia chromosomenegative ALL. Furthermore, the optimal use of VSLI remains to be defined. Possessing a clear advantage over conventional vincristine, VSLI is likely to find its optimal role in combination chemotherapy regimens, where it can be substituted for 
conventional vincristine in earlier phases of the disease. To this end, a randomized Phase III study to evaluate the substitution of VSLI for standard vincristine in the treatment of patients 60 years or older with newly diagnosed Philadelphia chromosome-negative ALL is about to open. The trial will compare VSLI with free vincristine in the standard CALGB 8811 regimen ${ }^{21}$ with the study arm incorporating VSLI dosed at $2.25 \mathrm{mg} / \mathrm{m}^{2}$ without a dose cap, with overall survival as the primary endpoint. ${ }^{86}$ By comparing the overall survival as well as neurotoxicity profile between the two arms in this study, the suggested benefits of greater efficacy with less toxicity of liposome-encapsulated vincristine observed in earlier studies will hopefully be confirmed.

While VSLI represents an important advance in the treatment of patients with relapsed ALL, the response rate and duration of response remain modest, with much room remaining for improvement. Future improvements in its liposomal formulation may allow greater delivery of vincristine to leukemic cells. For example, increasing the drugto-lipid ratio in sphingomyelin/cholesterol liposomes has been associated with enhanced antitumor activity in animal models of human cancers by further prolonging the half-life of vincristine. ${ }^{87}$ The use of a more saturated phospholipid, dihydrosphingomyelin, in sphingomyelin/cholesterol liposomes may further improve vincristine retention and result in a longer circulation half-life with potentially improved antitumor efficacy ${ }^{88}$ Finally, applying a calcium phosphate nanoshell to the liposome has resulted in higher cellular uptake in tumor cells compared with uptake from uncoated liposomes, attributed to improved drug release at the $\mathrm{pH}$ of the tumor interstitium. ${ }^{89}$ However, beyond improved delivery, future dosing recommendations for all vincristine formulations should take into account differences in drug metabolism in different patient populations. For example, patients who express the CYP3A5 genotype, found in 70\% of African Americans, suffer less neurotoxicity following treatment with vincristine, ${ }^{90}$ and therefore may be able to tolerate higher doses of the drug, even when encapsulated in liposomes. Finally, it is clear that all drug resistance in ALL will not be overcome simply by enhanced drug delivery or dose optimization of vincristine, and further investigation of novel agents and approaches are still urgently required to improve the outcome of ALL.

\section{Disclosure}

The authors report no competing financial interests in this work.

\section{References}

1. Jain N, Gurbuxani C, Rhee C, Stock W. Acute lymphoblastic leukemia in adults. In: Hoffman R, Benz EJ, Silberstein LE, Heslop HE, Weitz JI, Anastasi J, editors. Hematology: Basic Principles and Practice. 6th ed. Philadelphia, PA: Elsevier Saunders; 2013.

2. Dores GM, Devesa SS, Curtis RE, Linet MS, Morton LM. Acute leukemia incidence and patient survival among children and adults in the United States, 2001-2007. Blood. 2012;119(1):34-43.

3. Pui CH, Robison LL, Look AT. Acute lymphoblastic leukaemia. Lancet. 2008;371(9617):1030-1043.

4. Moorman AV, Chilton L, Wilkinson J, Ensor HM, Bown N, Proctor SJ. A population-based cytogenetic study of adults with acute lymphoblastic leukemia. Blood. 2010;115(2):206-214.

5. O'Brien S, Thomas D, Ravandi F, et al. Outcome of adults with acute lymphocytic leukemia after second salvage therapy. Cancer. 2008;113(11):3186-3191.

6. Thomas DA, Kantarjian H, Smith TL, et al. Primary refractory and relapsed adult acute lymphoblastic leukemia: characteristics, treatment results, and prognosis with salvage therapy. Cancer. 1999;86(7): 1216-1230.

7. Forman SJ, Rowe JM. The myth of the second remission of acute leukemia in the adult. Blood. 2013;121(7):1077-1082.

8. Hunger SP, Lu X, Devidas M, et al. Improved survival for children and adolescents with acute lymphoblastic leukemia between 1990 and 2005: a report from the Children's Oncology Group. J Clin Oncol. 2012;30(14):1663-1669.

9. Litzow MR. Evolving paradigms in the therapy of Philadelphiachromosome-negative acute lymphoblastic leukemia in adults. Hematology Am Soc Hematol Educ Program. 2009:362-370.

10. Hoelzer D, Thiel E, Loffler H, et al. Prognostic factors in a multicenter study for treatment of acute lymphoblastic leukemia in adults. Blood. 1988;71(1):123-131.

11. Hussein KK, Dahlberg S, Head D, et al. Treatment of acute lymphoblastic leukemia in adults with intensive induction, consolidation, and maintenance chemotherapy. Blood. 1989;73(1):57-63.

12. Fielding AK, Richards SM, Chopra R, et al. Outcome of 609 adults after relapse of acute lymphoblastic leukemia (ALL); an MRC UKALL12/ ECOG 2993 study. Blood. 2007;109(3):944-950.

13. Kako S, Kanamori H, Kobayashi N, et al. Outcome after first relapse in adult patients with Philadelphia chromosome-negative acute lymphoblastic leukaemia. Br J Haematol. 2013;161(1):95-103.

14. Gee TS, Haghbin M, Dowling MD, Cunningham I, Middleman MP, Clarkson BD. Acute lymphoblastic leukemia in adults and children. Differences in response with similar therapeutic regimens. Cancer. 1976;37(3):1256-1264.

15. Mayer RJ. Acute lymphoblastic leukemia in adults. Ann Intern Med. 1984;101(4):552-554.

16. Willemze R, Hillen H, Hartgrink-Groeneveld CA, Haanen C. Treatment of acute lymphoblastic leukemia in adolescents and adults: a retrospective study of 41 patients (1970-1973). Blood. 1975;46(6):823-834.

17. Chang HI, Yeh MK. Clinical development of liposome-based drugs: formulation, characterization, and therapeutic efficacy. Int $J$ Nanomedicine. 2012;7:49-60.

18. Bangham AD, Horne RW. Negative staining of phospholipids and their structural modification by surface-active agents in the electron microscope. J Mol Biol. 1964;8:660-668.

19. Carbone PP, Bono V, Frei E 3rd, Brindley CO. Clinical studies with vincristine. Blood. 1963;21:640-647.

20. Kantarjian HM, O'Brien S, Smith TL, et al. Results of treatment with hyper-CVAD, a dose-intensive regimen, in adult acute lymphocytic leukemia. J Clin Oncol. 2000;18(3):547-561.

21. Larson RA, Dodge RK, Burns CP, et al. A five-drug remission induction regimen with intensive consolidation for adults with acute lymphoblastic leukemia: Cancer and Leukemia Group B study 8811. Blood. 1995;85(8):2025-2037. 
22. Linker CA, Levitt LJ, O’Donnell M, Forman SJ, Ries CA. Treatment of adult acute lymphoblastic leukemia with intensive cyclical chemotherapy: a follow-up report. Blood. 1991;78(11):2814-2822.

23. Lamanna N, Heffner LT, Kalaycio M, et al. Treatment of adults with acute lymphoblastic leukemia: do the specifics of the regimen matter? Results from a prospective randomized trial. Cancer. 2013;119(6): 1186-1194.

24. Millar JL, Millar BC, Powles RL, et al. Liposomal vincristine for the treatment of human acute lymphoblastic leukaemia in severe combined immunodeficient (SCID) mice. Br J Haematol. 1998;102(3): 718-721.

25. Owellen RJ, Hartke CA, Dickerson RM, Hains FO. Inhibition of tubulinmicrotubule polymerization by drugs of the vinca alkaloid class. Cancer Res. 1976;36(4):1499-1502.

26. Sarris AH, Hagemeister F, Romaguera J, et al. Liposomal vincristine in relapsed non-Hodgkin's lymphomas: early results of an ongoing phase II trial. Ann Oncol. 2000;11(1):69-72.

27. Safinya CR, Ewert KK. Materials chemistry: Liposomes derived from molecular vases. Nature. 2012;489(7416):372-374.

28. Foa R, Vitale A, Vignetti M, et al. Dasatinib as first-line treatment for adult patients with Philadelphia chromosome-positive acute lymphoblastic leukemia. Blood. 2011;118(25):6521-6528.

29. Yanada M, Takeuchi J, Sugiura I, et al. High complete remission rate and promising outcome by combination of imatinib and chemotherapy for newly diagnosed BCR-ABL-positive acute lymphoblastic leukemia: a phase II study by the Japan Adult Leukemia Study Group. J Clin Oncol. 2006;24(3):460-466

30. Ribera JM, Garcia O, Fernandez-Abellan P, et al. Lack of negative impact of Philadelphia chromosome in older patients with acute lymphoblastic leukaemia in the thyrosine kinase inhibitor era: comparison of two prospective parallel protocols. Br J Haematol. 2012;159(4): 485-488.

31. Towatari M, Yanada M, Usui N, et al. Combination of intensive chemotherapy and imatinib can rapidly induce high-quality complete remission for a majority of patients with newly diagnosed BCR-ABL-positive acute lymphoblastic leukemia. Blood. 2004;104(12):3507-3512.

32. Goldstone AH, Richards SM, Lazarus HM, et al. In adults with standardrisk acute lymphoblastic leukemia, the greatest benefit is achieved from a matched sibling allogeneic transplantation in first complete remission, and an autologous transplantation is less effective than conventional consolidation/maintenance chemotherapy in all patients: final results of the International ALL Trial (MRC UKALL XII/ECOG E2993). Blood. 2008;111(4):1827-1833.

33. Duval M, Klein JP, He W, et al. Hematopoietic stem-cell transplantation for acute leukemia in relapse or primary induction failure. J Clin Oncol. 2010;28(23):3730-3738.

34. Kantarjian HM, Thomas D, Ravandi F, et al. Defining the course and prognosis of adults with acute lymphocytic leukemia in first salvage after induction failure or short first remission duration. Cancer. 2010;116(24):5568-5574.

35. Oliansky DM, Larson RA, Weisdorf D, et al. The role of cytotoxic therapy with hematopoietic stem cell transplantation in the treatment of adult acute lymphoblastic leukemia: update of the 2006 evidence-based review. Biol Bone Marrow Transplant. 2012;18(1):18-36. e6.

36. Marks DI. Treating the "older" adult with acute lymphoblastic leukemia. Hematology Am Soc Hematol Educ Program. 2010;2010:13-20.

37. Garcia-Manero G, Thomas DA. Salvage therapy for refractory or relapsed acute lymphocytic leukemia. Hematol Oncol Clin North Am. 2001;15(1):163-205.

38. Meyer JA, Wang J, Hogan LE, et al. Relapse-specific mutations in NT5C2 in childhood acute lymphoblastic leukemia. Nat Genet. 2013;45(3):290-294.

39. Tzoneva G, Perez-Garcia A, Carpenter Z, et al. Activating mutations in the $\mathrm{NT} 5 \mathrm{C} 2$ nucleotidase gene drive chemotherapy resistance in relapsed ALL. Nat Med. 2013;19(3):368-371.
40. Uckun FM, Qazi S, Ozer Z, et al. Inducing apoptosis in chemotherapyresistant B-lineage acute lymphoblastic leukaemia cells by targeting HSPA5, a master regulator of the anti-apoptotic unfolded protein response signalling network. Br J Haematol. 2011;153(6):741-752.

41. Pramanik R, Sheng X, Ichihara B, Heisterkamp N, Mittelman SD. Adipose tissue attracts and protects acute lymphoblastic leukemia cells from chemotherapy. Leuk Res. 2013;37(5):503-509.

42. Litzow MR. Novel therapeutic approaches for acute lymphoblastic leukemia. Hematol Oncol Clin North Am. 2011;25(6):1303-1317.

43. Mathisen MS, Jabbour E, Kantarjian HM. Treatment of adult acute lymphoblastic leukemia (ALL) with a focus on emerging investigational and targeted therapies. Oncology (Williston Park). 2012;26(9):851-859.

44. Hoelzer D. Treatment of acute lymphoblastic leukemia. Semin Hematol. 1994;31(1):1-15.

45. Gabizon AA. Selective tumor localization and improved therapeutic index of anthracyclines encapsulated in long-circulating liposomes. Cancer Res. 1992;52(4):891-896.

46. Forssen EA, Coulter DM, Proffitt RT. Selective in vivo localization of daunorubicin small unilamellar vesicles in solid tumors. Cancer Res. 1992;52(12):3255-3261.

47. Mayer LD, Bally MB, Loughrey H, Masin D, Cullis PR. Liposomal vincristine preparations which exhibit decreased drug toxicity and increased activity against murine L1210 and P388 tumors. Cancer Res. 1990;50(3):575-579.

48. Mayer LD, Nayar R, Thies RL, Boman NL, Cullis PR, Bally MB. Identification of vesicle properties that enhance the antitumour activity of liposomal vincristine against murine L1210 leukemia. Cancer Chemother Pharmacol. 1993;33(1):17-24.

49. Mayer LD, St-Onge G. Determination of free and liposome-associated doxorubicin and vincristine levels in plasma under equilibrium conditions employing ultrafiltration techniques. Anal Biochem. 1995;232(2): 149-157.

50. Perez-Soler R, Han I, al-Baker S, Khokhar AR. Lipophilic platinum complexes entrapped in liposomes: improved stability and preserved antitumor activity with complexes containing linear alkyl carboxylato leaving groups. Cancer Chemother Pharmacol. 1994;33(5):378-384.

51. Boman NL, Bally MB, Cullis PR, Mayer LD, Webb MS. Encapsulation of vincristine in liposomes reduces toxicity and improves its anti-tumor efficacy. J Liposome Res. 1995;5(3):523-541.

52. Gabizon A, Catane R, Uziely B, et al. Prolonged circulation time and enhanced accumulation in malignant exudates of doxorubicin encapsulated in polyethylene-glycol coated liposomes. Cancer Res. 1994;54(4): 987-992.

53. Campbell RB, Ying B, Kuesters GM, Hemphill R. Fighting cancer: from the bench to bedside using second generation cationic liposomal therapeutics. J Pharm Sci. 2009;98(2):411-429.

54. Krishna R, Webb MS, St Onge G, Mayer LD. Liposomal and nonliposomal drug pharmacokinetics after administration of liposomeencapsulated vincristine and their contribution to drug tissue distribution properties. J Pharmacol Exp Ther. 2001;298(3):1206-1212.

55. O'Brien ME, Wigler N, Inbar M, et al. Reduced cardiotoxicity and comparable efficacy in a phase III trial of pegylated liposomal doxorubicin $\mathrm{HCl}$ (CAELYX/Doxil) versus conventional doxorubicin for first-line treatment of metastatic breast cancer. Ann Oncol. 2004;15(3): 440-449.

56. Hussein MA, Wood L, Hsi E, et al. A Phase II trial of pegylated liposomal doxorubicin, vincristine, and reduced-dose dexamethasone combination therapy in newly diagnosed multiple myeloma patients. Cancer. 2002;95(10):2160-2168.

57. Cortes J, O'Brien S, Estey E, Giles F, Keating M, Kantariian H. Phase I study of liposomal daunorubicin in patients with acute leukemia. Invest New Drugs. 1999;17(1):81-87.

58. Gill PS, Wernz J, Scadden DT, et al. Randomized phase III trial of liposomal daunorubicin versus doxorubicin, bleomycin, and vincristine in AIDS-related Kaposi's sarcoma. J Clin Oncol. 1996;14(8): 2353-2364. 
59. Glantz MJ, LaFollette S, Jaeckle KA, et al. Randomized trial of a slowrelease versus a standard formulation of cytarabine for the intrathecal treatment of lymphomatous meningitis. J Clin Oncol. 1999;17(10): 3110-3116.

60. Feldman EJ, Lancet JE, Kolitz JE, et al. First-in-man study of CPX-351: a liposomal carrier containing cytarabine and daunorubicin in a fixed 5:1 molar ratio for the treatment of relapsed and refractory acute myeloid leukemia. J Clin Oncol. 2011;29(8):979-985.

61. Feldman EJ, Kolitz JE, Trang JM, et al. Pharmacokinetics of CPX-351; a nano-scale liposomal fixed molar ratio formulation of cytarabine:daunorubicin, in patients with advanced leukemia. Leuk Res. 2012;36(10):1283-1289.

62. Owellen RJ, Root MA, Hains FO. Pharmacokinetics of vindesine and vincristine in humans. Cancer Res. 1977;37(8 Pt 1):2603-2607.

63. Sethi VS, Jackson DV Jr, White DR, et al. Pharmacokinetics of vincristine sulfate in adult cancer patients. Cancer Res. 1981;41(9 Pt 1): 3551-3555.

64. Haim N, Epelbaum R, Ben-Shahar M, Yarnitsky D, Simri W, Robinson E. Full dose vincristine (without 2-mg dose limit) in the treatment of lymphomas. Cancer. 1994;73(10):2515-2519.

65. Layton D, Trouet A. A comparison of the therapeutic effects of free and liposomally encapsulated vincristine in leukemic mice. Eur J Cancer. 1980;16(7):945-950.

66. Mayer LD, Tai LC, Ko DS, et al. Influence of vesicle size, lipid composition, and drug-to-lipid ratio on the biological activity of liposomal doxorubicin in mice. Cancer Res. 1989;49(21):5922-5930.

67. Bally MB, Mayer LD, Loughrey H, et al. Dopamine accumulation in large unilamellar vesicle systems induced by transmembrane ion gradients. Chem Phys Lipids. 1988;47(2):97-107.

68. Mayer LD, Wong KF, Menon K, Chong C, Harrigan PR, Cullis $P R$. Influence of ion gradients on the transbilayer distribution of dibucaine in large unilamellar vesicles. Biochemistry. 1988;27(6): 2053-2060.

69. Boman NL, Masin D, Mayer LD, Cullis PR, Bally MB. Liposomal vincristine which exhibits increased drug retention and increased circulation longevity cures mice bearing P388 tumors. Cancer Res. 1994;54(11):2830-2833.

70. Webb MS, Harasym TO, Masin D, Bally MB, Mayer LD. Sphingomyelin-cholesterol liposomes significantly enhance the pharmacokinetic and therapeutic properties of vincristine in murine and human tumour models. Br J Cancer. 1995;72(4):896-904.

71. Allen TM, Hansen C. Pharmacokinetics of stealth versus conventional liposomes: effect of dose. Biochim Biophys Acta. 1991;1068(2): 133-141.

72. Blume G, Cevc G. Molecular mechanism of the lipid vesicle longevity in vivo. Biochim Biophys Acta. 1993;1146(2):157-168.

73. Wong M, Kantarjian H, Appelbaum F, et al. Vincristine sulfate liposomes injection concentrates vincristine in tumor tissue and bone marrow of tumor-bearing mice. American Association for Cancer Research National Cancer Institute - European Organisation for Research and Treatment of Cancer: International Conference on Molecular Targets and Cancer Therapeutics; October 22-26, 2007; San Francisco, CA, USA. Poster Presentation C111.

74. Kanter PM, Klaich GM, Bullard GA, King JM, Bally MB, Mayer LD. Liposome encapsulated vincristine: preclinical toxicologic and pharmacologic comparison with free vincristine and empty liposomes in mice, rats and dogs. Anticancer Drugs. 1994;5(5):579-590.
75. Leonetti C, Scarsella M, Semple SC, et al. In vivo administration of liposomal vincristine sensitizes drug-resistant human solid tumors. Int J Cancer. 2004;110(5):767-774.

76. Silverman JA, Deitcher SR. Marqibo ${ }^{\circledR}$ (vincristine sulfate liposome injection) improves the pharmacokinetics and pharmacodynamics of vincristine. Cancer Chemother Pharmacol. 2013;71(3):555-564.

77. Gelmon KA, Tolcher A, Diab AR, et al. Phase I study of liposomal vincristine. J Clin Oncol. 1999;17(2):697-705.

78. Embree L, Gelmon K, Tolcher A, et al. Pharmacokinetic behavior of vincristine sulfate following administration of vincristine sulfate liposome injection. Cancer Chemother Pharmacol. 1998;41(5): 347-352.

79. Thomas DA, Sarris AH, Cortes J, et al. Phase II study of sphingosomal vincristine in patients with recurrent or refractory adult acute lymphocytic leukemia. Cancer. 2006;106(1):120-127.

80. Thomas DA, Kantariian HM, Stock W, et al. Phase 1 multicenter study of vincristine sulfate liposomes injection and dexamethasone in adults with relapsed or refractory acute lymphoblastic leukemia. Cancer. 2009;115(23):5490-5498

81. O'Brien S, Schiller G, Lister J, et al. High-dose vincristine sulfate liposome injection for advanced, relapsed, and refractory adult Philadelphia chromosome-negative acute lymphoblastic leukemia. J Clin Oncol. 2013;31(6):676-683.

82. Deitcher OR, O’Brien S, Deitcher SR, Thomas DA, Kantarjian HM. Single-agent vincristine sulfate liposomes injection (Marqibo ${ }^{\circledR}$ ) compared to historical single-agent therapy for adults with advanced, relapsed and/or refractory Philadelphia chromosome negative acute lymphoblastic leukemia. ASH Annual Meeting Abstracts. 2011;118(21): 2592.

83. Kantarjian H, Gandhi V, Cortes J, et al. Phase 2 clinical and pharmacologic study of clofarabine in patients with refractory or relapsed acute leukemia. Blood. 2003;102(7):2379-2386.

84. Advani AS, Gundacker HM, Sala-Torra O, et al. Southwest Oncology Group Study S0530: a phase 2 trial of clofarabine and cytarabine for relapsed or refractory acute lymphocytic leukaemia. Br J Haematol. 2010;151(5):430-434.

85. Cohen MH, Johnson JR, Justice R, Pazdur R. FDA drug approval summary: nelarabine (Arranon) for the treatment of T-cell lymphoblastic leukemia/lymphoma. Oncologist. 2008;13(6):709-714.

86. Deitcher SR, Silverman JA. Design of the Hallmarq trial, a Phase 3 study of vincristine sulfate liposome injection (VSLI, Marqibo ${ }^{\circledR}$ ) in adults with newly diagnosed, Philadelphia chromosome negative acute lymphoblastic leukemia. ASH Annual Meeting Abstracts. 2012;120(21):4300.

87. Johnston MJ, Semple SC, Klimuk SK, et al. Therapeutically optimized rates of drug release can be achieved by varying the drug-to-lipid ratio in liposomal vincristine formulations. Biochim Biophys Acta. 2006;1758(1):55-64.

88. Johnston MJ, Semple SC, Klimuk SK, Ansell S, Maurer N, Cullis PR. Characterization of the drug retention and pharmacokinetic properties of liposomal nanoparticles containing dihydrosphingomyelin. Biochim Biophys Acta. 2007;1768(5):1121-1127.

89. Thakkar HP, Baser AK, Parmar MP, Patel KH, Ramachandra Murthy R. Vincristine-sulphate-loaded liposome-templated calcium phosphate nanoshell as potential tumor-targeting delivery system. $J$ Liposome Res. 2012;22(2):139-147.

90. Egbelakin A, Ferguson MJ, MacGill EA, et al. Increased risk of vincristine neurotoxicity associated with low CYP3A5 expression genotype in children with acute lymphoblastic leukemia. Pediatr Blood Cancer. 2011;56(3):361-367.
International Journal of Nanomedicine

\section{Publish your work in this journal}

The International Journal of Nanomedicine is an international, peerreviewed journal focusing on the application of nanotechnology in diagnostics, therapeutics, and drug delivery systems throughout the biomedical field. This journal is indexed on PubMed Central, MedLine, CAS, SciSearch ${ }^{\circledR}$, Current Contents ${ }^{\circledR} /$ Clinical Medicine,

\section{Dovepress}

Journal Citation Reports/Science Edition, EMBase, Scopus and the Elsevier Bibliographic databases. The manuscript management system is completely online and includes a very quick and fair peer-review system, which is all easy to use. Visit http://www.dovepress.com/ testimonials.php to read real quotes from published authors. 